\title{
Psychological and Physical Impacts of Extreme Events on Older Adults: Implications for Communications
}

Erin McClelland, MA; Richard Amlôt, PhD; M. Brooke Rogers, PhD; G. James Rubin, PhD; John Tesh, BA (Hons); Julia M. Pearce, PhD

\section{ABSTRACT}

In recent years, a series of large-scale, high-profile natural disasters and terrorist attacks have demonstrated the need for thorough and effective disaster preparedness. While these extreme events affect communities and societies as a whole, they also carry specific risks for particular population groups. Crises such as Hurricane Katrina and the 2011 earthquake and tsunami disaster in Japan have illustrated the risk of significant and disproportionate morbidity and mortality among older adults during disasters. Age does not necessarily equate to vulnerability, but many physical and psychological consequences of the aging process can increase the risk of adverse outcomes. As the older population grows, so too does the need to ensure that adequate, practical, and appropriate measures exist to offset the specific risks from extreme events associated with this subpopulation. Effective risk and crisis communication plays a key role in mitigating the extent to which older adults are differentially affected during extreme events. By identifying the specific issues affecting older adults, this review highlights important areas for action for practitioners and policy-makers, particularly in the realm of crisis communication. (Disaster Med Public Health Preparedness. 2017;11:127-134)

Key Words: vulnerable populations, communication, disasters

$\mathrm{D}$ isasters and public health events broadly affect communities and societies as a whole, but they also carry specific risks and outcomes for particular population groups. These high-risk groups have been categorized in a variety of ways, including by geography, ${ }^{1}$ ethnicity, ${ }^{2}$ and age. ${ }^{3}$ Older adults in particular are frequently highlighted as a subpopulation requiring specific attention or care. The 2008 UK Cabinet Office report "Identifying People Who Are Vulnerable in a Crisis" recognizes 13 vulnerable groups including older people, ${ }^{4}$ and the United Kingdom's national adaptation plan for climate change has a particular focus on measures to mitigate the expected effects of changes in the climate on these groups, and in particular the elderly. ${ }^{5}$ The US Department of Health and Human Services lists older adults among the special populations for emergency preparedness and disaster. ${ }^{6}$ In addition, the World Health Organization (WHO) recognizes older individuals as a vulnerable group in emergencies. ${ }^{7}$ Although various reports and agencies categorize older adults differently, definitions usually restrict the group to a minimum age of between 60 and 65 years.

As witnessed during Hurricane Katrina and the 2011 earthquake in Japan, older adults suffer from increased morbidity and mortality rates. Prior to Hurricane Katrina, individuals over the age of 60 years represented $15 \%$ of the population of New Orleans; however, this group accounted for approximately $75 \%$ of the bodies found. ${ }^{8}$ Following the Great East Japan Earthquake in March 2011, an estimated 95\% of those who perished were above age $60 .{ }^{9}$ That older adults appear to be disproportionately affected by disasters not only represents an area of humanitarian concern, but also highlights the challenges that responding organizations face as they work to support affected populations.

It is possible that older adults also suffer disproportionately from the secondary effects of major disasters. These are defined, in UK law for example, as effects which result from the primary hazard and significantly impact human welfare, including homelessness (in the sense of a need for evacuation from an individual's customary place of residence) and damage to property; disruption of a supply of money, food, water, energy, or fuel; and disruption of facilities for transport or services relating to health. ${ }^{10}$ The need to pay attention to these secondary impacts on essential services has been accentuated in recent years because they are increasingly interlinked so that disruptions in one tend to result in disruptions to others.

Aside from the moral implications of meeting the needs of the vulnerable in our societies, the proportion of older adults in society is growing. For example, in the United Kingdom the percentage of the 
population over the age of 65 years is expected to increase from 1 in 6 to 1 in 4 by 2050. ${ }^{11}$ The 2001 United Nations "World Population Ageing: 1950-2050" report noted that this move toward an aging population is a global development. In 1950 the proportion of older adults was 8\%, increasing in 2000 to $10 \%$ and expected to rise as high as $21 \%$ by $2050 .{ }^{12}$ As the older population grows, so too does the need to ensure that adequate, practical, and appropriate measures are in place to offset the specific risks of extreme events within this subpopulation. Increased vulnerability of older adults during extreme events is already recognized as a matter of policy in many areas. Seasonal vaccine priority lists include older adults as a target category, ${ }^{13}$ and heat wave preparedness identifies older adults as an at-risk group. ${ }^{14}$ However, this approach and its implementation needs to be reviewed for extreme events more broadly, particularly in the realm of crisis communication.

The importance of promoting a culture of resilience and supporting community disaster resilience-ie, the ability of communities to withstand, respond to, and recover from extreme events-is increasingly recognized. ${ }^{15,16}$ The promotion of resilience for older adults can be achieved by having clear and open responsibilities for risk-related decisionmaking; rehearsing adaptable crisis management arrangements, with good communication across boundaries; having a good understanding of the risks of emergencies and how they can impact different groups; and, potentially most importantly, communicating a common understanding of risk in order to mobilize responsible individuals and organizations to invest in preparedness. ${ }^{15}$ Fostering resilience better prepares individuals, including potentially vulnerable groups like older adults, to manage risks. ${ }^{17}$

This article examines the impacts of extreme events on older adults and focuses on how effective communication can improve health outcomes. It considers to what extent approaches to risk communication need to reflect the differences between this group and the rest of the population in terms of their abilities to cope with the psychological impacts (where older people may in fact be more robust than younger people) and the physiological impacts (where the opposite is more likely to be true). The usefulness of communication to offset extreme events is considered at a variety of stages including the pre-event stage (risk communication as education), the alerting stage (warning), the post-onset stage (informing decisions during an ongoing crisis), and the postevent stage (including recovery).

We identified primary sources for this review by searching electronic databases (Web of Science [Thomson Reuters] and Ovid [Wolters Kluwer]) by using combinations of search terms including "old*," "senior," "elder*," "disaster," "hurricane," "earthquake," "tsunami," "terror*," "behav*," "evacuate," "information-seeking," and "shelter." This search was limited to English-language documents. This resulted in
1804 articles identified (excluding duplicates), of which 1563 were eliminated on the basis of relevance or access, leaving 241 articles for full-text review. A further search was conducted focused on communication using combinations of search terms including "old*," "senior," "elder," "disaster," "hurricane," "earthquake," "tsunami," "terror*," and "communicat*." This resulted in 239 articles identified (excluding duplicates), of which 235 were eliminated on the basis of relevance, access, or duplication with the initial search leaving an additional 4 articles for full-text review. This formal review process was supplemented with articles and policy documents identified by subject matter experts, the reference lists of included papers, and the websites of organizations such as Public Health England and the WHO. This produced a total sample of 267 documents for full-text review. These sources were coded thematically by using NVivo (QSR International) to identify the physical and mental health impacts of extreme events on older adults and the role of communication in offsetting these outcomes.

\section{OLDER ADULTS AND EXTREME EVENTS Age Alone Does Not Determine Outcome}

Although older adults represent a higher risk category in disaster outcomes, it is important to recognize that age, in and of itself, is not necessarily problematic but represents a state that tends to be paired with, and indeed often results in, other characteristics that render an older person more vulnerable. These include "loss of skeletal muscle and strength, reduced bone mass, hearing loss and decreased visual acuity" ${ }^{18}$ as well as chronic health conditions. ${ }^{19}$ These health challenges, combined with the natural development of old age, can be problematic when preparing disaster mitigation strategies. For example, as a consequence of diminishing visual capacity, an individual may lose the ability to drive and may thus require higher levels of aid to evacuate or to receive necessary supplies such as medication. ${ }^{20}$

Whereas these conditions may render an individual more vulnerable, in a day-to-day context many of the difficulties can be mitigated with support such as care workers or public transportation networks. In a disaster, these aids may not be available, resulting in a situation-specific increased rate of vulnerability. For example, in the 2006 war between Israel and Lebanon, many homecare workers stayed in their homes, which left the individuals (primarily disabled adults) reliant on them without food, medicine, or care. Consequently, this subset of the older population was at greater risk of harm due to mobility issues and the often rapid need to take shelter. ${ }^{21}$ This interdependent dynamic of emergency circumstances and vulnerable characteristics is highlighted in the literature, recognizing that, in addition to health challenges, older adults are more likely to have logistical and social difficulties such as mobility issues, lack of transportation, and limited social networks. ${ }^{22}$ Consequently, researchers have proposed the idea of cumulative risk as a stronger predictor of 
resilience. This suggests that a variety of risks (including age) combine to determine the level of resilience. ${ }^{23}$

It is necessary to consider subpopulations, such as older adults, as a whole when trying to determine a baseline for average capabilities and requirements. It is, however, also important to note the valuable contribution that older adults can make, as well as the level of resilience many of them possess. Older adults do tend to be more vulnerable to disaster and are disproportionately represented in morbidity and mortality rates, ${ }^{24}$ but the very nature of their having reached advanced years demonstrates that they are also survivors. Although planning must take into account the needs of the more vulnerable among this population, many are as capable or more so than the average citizen, and age should not be considered to equate to liability in this context. Older adults tend to be retirees and are often very active in their communities and families. They disproportionately include the volunteers who help mail letters, answer phones on political campaigns, and watch the grandchildren while parents are at work. Resilient older adults can also serve as resources to help younger populations understand how to cope with stressful situations. ${ }^{23}$ Recognizing and valuing these resilience attributes alongside the potential for older adults to be more vulnerable to disasters will aid the development of adequate, practical, and appropriate measures designed to harness capabilities and offset the specific risks of extreme events associated with this subpopulation.

\section{Psychological Effects}

Despite a large body of research which has assessed the psychological vulnerability of older populations to extreme events, little agreement exists regarding the proportional level of psychological effects, such as post-traumatic stress disorder and depression, suffered by older adult disaster survivors. Some researchers have posited that older adults experience greater psychological distress, ${ }^{25}$ others that there is no marked difference between older adults and the general population, ${ }^{26}$ and still others maintain that the level of psychological stress experienced by older adults is in fact lower than average. ${ }^{27}$ This range of outcomes has been attributed to a number of factors such as the socioeconomics of the countries affected, ${ }^{28}$ varied definitions of older adults, ${ }^{29}$ type of assessment, duration of time examined, and intensity of the event. ${ }^{30}$

Several theories have been used to interpret the various study outcomes and, as with the studies themselves, these theoretical approaches highlight differences in the characterization of older adults' ability to cope with extreme events and disasters. For example, the inoculation hypothesis posits that older adults are better able to respond to crisis because they have likely dealt with severe challenges in the past, ${ }^{31}$ whereas the maturation hypothesis proposes that successful aging brings with it a level of resilience due to more successful coping strategies. ${ }^{32}$ A study testing the 2 theories in relation to older adults' responses to the 2001 World Trade Center attacks and to Hurricane Sandy found support for the maturation hypothesis in the case of Hurricane Sandy and for the inoculation hypothesis when examining the 2001 attacks. ${ }^{33}$ On the other hand, the differential vulnerability hypothesis suggests that the burden of coping with the challenges of aging (physical vulnerabilities, loss of loved ones, etc), the potential for financial difficulty, and years of coping in response to challenges can limit the ability of older people to deal with an extreme event. ${ }^{28}$ This theory would help to explain the studies indicating increased negative psychological outcomes for older adults following an extreme event.

Although there is disagreement on the extent, it is clear that older populations do suffer from adverse psychological effects in the wake of a disaster. In addition, there is evidence to suggest that older people may be less likely to access postdisaster services, including but not limited to psychological assistance. $^{25,34}$ This has implications for planning and in particular for communication, as it becomes necessary not only to attempt to identify and triage older patients in disaster shelters and other locations, but also to ensure that older adults are aware of the psychological services and benefits available to them. It is also important that these are presented in a manner that encourages uptake, because older adults have been shown to be less likely than their younger counterparts to complain or to ask for support. ${ }^{35}$

While exposure to past trauma may render older populations more impervious to the psychological effects of extreme events, there is also evidence to suggest that this may make them less likely to accept warnings. ${ }^{34}$ Many older adults have chosen not to evacuate during hurricanes because they had survived previous incidents unscathed or evacuated unnecessarily in the past and so chose not to heed the present warnings. ${ }^{36}$ Furthermore, disasters requiring evacuation or relocation can cause additional stress and mental health issues, with displaced older adults suffering greater psychological strain than those able to remain resident. ${ }^{37}$ A 2013 US study found that, despite being at greater risk due to disruptions in infrastructure and support, older populations were inclined to shelter in place rather than evacuate. ${ }^{38}$ There are several ways in which this issue could be addressed, for instance, encouraging greater personal preparedness and ensuring that emergency preparedness plans (at all levels of government) take into account the needs of the older population. However, reexamining emergency communications-both messaging and medium-from a vulnerable populations perspective may help to provide suggestions for improving older adults' uptake of protective behaviors, such as evacuation, in the future.

\section{Physiological Effects}

Unlike psychological impacts, there is clear evidence that older adults are disproportionately physiologically affected by 
disasters. While a small number of studies have demonstrated a reduced likelihood of adverse physiological effects, these have been linked to very specific circumstances. For example, a number of studies on flooding have indicated the most vulnerable group are middle-aged men, but this is attributed to risky behavior such as attempting to swim across rivers in floods. ${ }^{39}$ The overwhelming majority of evidence indicates that in a disaster the mortality and morbidity rates among older populations are dramatically disproportionate to the general population. The high level of medical assistance needed by many vulnerable older persons and the already present physical challenges increase the likelihood of disproportionate casualty rates for this particular sector of society. ${ }^{40}$ For example, despite representing $12 \%$ to $15 \%$ of the affected population, $75 \%$ of deaths associated with Hurricane Katrina were among older populations. The adverse effects were not limited to mortality. A study of New Orleans Medicare Advantage Plan enrollees conducted 1 year after the hurricane also demonstrated higher morbidity compared to national rates. ${ }^{41}$

Aging often results in general health complications and many older adults suffer from chronic medical conditions requiring treatments either at home or by local health services that may themselves be disrupted during emergencies. This characteristic increases the likelihood of adverse physiological outcomes during crisis. For example, a study on the 2011 earthquake in Japan highlighted that an inability to obtain medicine for chronic conditions resulted in worsening health for many older individuals. ${ }^{42}$ Studies in developed countries indicate that up to $40 \%$ of individuals over 65 years of age suffer from a chronic illness or disability, less than one-third of adults over 75 years of age are in good health, and over one-third of adults over 80 years of age are unable to walk outside without assistance. ${ }^{43}$ This has serious implications for extreme events because mobility and health challenges may impede successful emergency management, in particular evacuation.

Nowhere have the specific adverse physiological effects of disasters on older adults been more clearly seen than in care homes, particularly in an evacuation setting. The decision to evacuate or shelter in place is driven by a number of factors, including the logistical challenge of transporting frail patients. Not only must transport be acquired but a safe destination that will meet the basic needs of the patients ideally confirmed. ${ }^{44}$ The 2011 post-earthquake Fukishima radiation incident resulted in a mandatory evacuation zone. Among the evacuees were 1770 residents of care homes and hospitals. The need to evacuate quickly resulted in patients being moved without medical care, blankets, or safety restraints. This resulted in adverse outcomes including traumatic injuries and death from dehydration and pneumonia. ${ }^{45}$ Furthermore, patients with dementia tend to be particularly badly affected by evacuation. A study of dementia patients evacuated for Hurricane Gustav indicated a 2.8\% mortality increase at 30 days and a $3.9 \%$ increase at 90 days when compared to dementia patients who did not evacuate. This increase in mortality may be due to existing health conditions being aggravated by the disruption of regular daily living schedules and being in unfamiliar surroundings. ${ }^{46}$

Greater morbidity and mortality among older populations is not restricted to natural disasters. Seasonal influenza targets the youngest (under 2 years of age) and oldest (over 65 years) of the population as well as those with specific medical conditions. ${ }^{47}$ These subpopulations not only account for the majority of influenza-related hospitalizations, but older people alone account for over $90 \%$ of influenza-related deaths, often as a result of complications that develop, in particular pneumonia. ${ }^{48}$ For those individuals over the age of 70 years, the risks of hospitalization and mortality increase. ${ }^{49}$ In addition, influenza infections in older individuals have also been associated with decreased overall health such as functional decline. ${ }^{50}$ Protective measures such as handwashing and vaccination can help to reduce the risk of infection. Vaccination, in particular, can prevent seasonal influenzaassociated fatalities in older populations by as much as $80 \% .^{50}$ Aging, however, often results in a reduction in immunity. This increases an individual's vulnerability not only to influenza but also to other infectious diseases and may limit the body's ability to respond as effectively to preventive treatments such as vaccinations. ${ }^{48}$

\section{THE USE OF RISK AND CRISIS COMMUNICATION TO SUPPORT BETTER OUTCOMES FOR VULNERABLE OLDER ADULTS}

Effective communication plays a key role in preparing for, responding to, and recovering from public health emergencies. $^{51,52}$ Communication can reduce uncertainty around an extreme event, providing reassurance and direction. ${ }^{53}$ Communication surrounding emergency events can be categorized as risk communication, crisis communication, and crisis and emergency risk communication. Risk communication is not limited to crises and can increase awareness in the population of risks including personal choices (such as diet). ${ }^{54}$ Crisis communication is much more specific, with a focus on "risks that are manifested." ${ }^{, 2,55,56}$ Crisis and emergency risk communication draws on the principles of both risk and crisis communication, as well as issues management, in order to successfully communicate with the public during an emergency situation. ${ }^{55}$

\section{Pre-Event Communication}

Pre-event communication campaigns can be useful in preparing populations for extreme events as well as encouraging protective behavior. Communication campaigns have been effectively designed for a number of different risks and threats. For example, communications focused on the needs of older adults, such as campaigns to promote vaccination ${ }^{57,58}$ and heat-related communications campaigns, ${ }^{59}$ have been 
successful in encouraging positive health behaviors. However, a study on preparedness indicated that two-thirds of older US adults did not have an emergency plan and had not taken part in any disaster preparedness education. ${ }^{60}$ This lack of preparedness among older adults is not just an American phenomenon. A 2012 study of older adult preparedness in Hong Kong found that, "preparedness of elderly people in the present study is considered grossly inadequate." ${ }^{61}$ Individual emergency preparedness is important and pre-event communication can be used to highlight this and encourage personal planning. Emergency preparedness planning measures such as education booklets and lists specific to the general circumstances and needs of the older population can help to improve the disaster readiness of older individuals. ${ }^{61}$

Development of disaster management guidelines requires consideration of specific circumstances involving older populations. This includes aspects such as mobility, general health, and cognitive challenges as well as emotional considerations. A report from the Centers for Disease Control and Prevention on disaster planning for older adults highlighted that some older adults are reluctant to leave behind a lifetime of possessions in order to evacuate. ${ }^{62}$ This advice is reinforced by a WHO report which identified that older adults may be reluctant to evacuate because it means leaving their "lifetime homes." ${ }^{43}$ Similarly, a study of older individuals in Georgia and North Carolina found that approximately $38 \%$ of pet-owning respondents either would not evacuate because of their pet or would only evacuate with their pet. ${ }^{63}$ It is important that communication is not unidirectional. Including older adults in the planning phase can result in a better understanding of the needs of this group and lead to improved emergency preparedness planning. ${ }^{41}$ For example, hearing firsthand about past experiences with disasters, what worked and what did not, can help to identify the specific challenges for older adults.

\section{Communication During an Event}

Effective communication during an extreme event can be used to raise awareness, improve knowledge, and reduce negative social, psychological, and health impacts. ${ }^{51,52,64}$ Conversely, lack of communication may exacerbate negative impacts of disasters and emergencies. For example, insufficient information during the rapid evacuation of care homes in response to the 2011 earthquake in Christchurch, New Zealand, resulted in confusion and anxiety among older adult residents. ${ }^{65}$ There are a number of well-established principles for effective communication during an emergency, namely, the provision of accurate, timely, consistent, clear, and simple-to-understand information. ${ }^{56,64,66}$ It is particularly important to avoid technical or complex language when communicating with older adults owing to low levels of health literacy in this group and age-related cognitive decline. ${ }^{67}$ For example, in the Veterans Affairs nursing homes affected by Hurricanes Katrina and Rita, an estimated 50\% of residents had some form of cognitive difficulty. As a result, staff in these homes took care to use easily understandable language to ensure that residents understood the situation. ${ }^{18}$

Crisis and emergency risk communication should also consider the mode of communication used to ensure that information reaches older adults. The move toward online information sources, while providing a rapid and costeffective way to deliver messaging, disadvantages those individuals who are not active online, do not have access to computers, and are not familiar with the Internet. The 2015 UK Internet Users study indicated that, while Internet use among older adults is increasing, $61 \%$ of adults over 75 years of age have never used the Internet. ${ }^{68}$ As a result, any web-based advice and warnings would not have the same reach in these subpopulations as messaging delivered through another medium. A study on public perception of severe weather warnings in Nova Scotia reinforces this conclusion. Older adult participants in the study were far more likely to receive news from television, radio, and nonmedia sources (ie, word of mouth), whereas younger participants favored the Internet. ${ }^{69}$ Research into responses to bioterrorism resulted in similar responses, with older individuals expressing intent to receive information from health care providers or emergency departments. ${ }^{70}$

\section{Post-Event Communication}

Clear and concise communication is not only necessary in pre-event planning and during an event, it is also required during the recovery period. For example, an assessment of older adults affected by flooding identified the need for "ordinary" language rather than technical terminology or bureaucrat-ese to encourage repair and rehabilitation. ${ }^{71}$ Post-event communication actions largely center on communicating lessons learned. ${ }^{72}$ There are, however, still avenues where event-related communication with the public is needed owing to secondary stressors such as economic challenges, health problems, the loss of personal possessions, and disruption of daily activities. ${ }^{73}$ For example, in the wake of Hurricane Katrina, it was noted that many older adults, particularly those without family or the wherewithal to navigate the bureaucratic requirements to obtain money to rebuild, simply "gave up." ${ }^{8}$ While this highlights the procedural challenges as much as the communication challenges, it does provide an opportunity to employ effective and targeted communication, not only to advise individuals of the support available but also to ensure that the steps involved in accessing support are laid out as clearly as possible. Older adults should not be burdened with the task of negotiating needlessly complex and verbose bureaucratic hurdles when recovering from a disaster.

\section{CONCLUSION}

Although some disagreement exists on the extent to which older adults are disproportionately affected by disasters, it is 
clear that this subpopulation is over-represented in overall mortality and morbidity rates. Including older individuals in the planning process, in particular, planning communications strategies, provides an opportunity to improve outcomes and better understand the needs of this community. The needs of this subgroup must be considered, integrated, and accommodated for in disaster planning and policy. This does not necessarily mean that separate plans are required, but rather that inclusive planning and preparedness recognize the distinct needs and challenges associated with this group. In particular, effective communication is essential, and messaging must be provided in a manner and medium appropriate for older adults.

Effective communication is a vital component of emergency planning and response to encourage personal preparedness, reduce uncertainty, and inform behavioral responses to extreme events. The requirement for clear and concise communication is not exclusive to older adults; however, this population group is likely to have specific needs that must be taken into account in emergency preparedness and response. This review was conducted to identify the impacts of extreme events on older adults to support the development of targeted crisis communication. This review suggests that while the impacts of extreme events on older adults have been well documented, the role of communication in mitigating these impacts has not received the same level of attention. In particular, there is a need for greater examination of specific communication guidance for older adults and empirically tested messaging.

Issues to consider for emergency planning:

- The specific needs, challenges, and requirements affecting older adults must be taken into account when developing emergency preparedness and communication plans in order to improve health outcomes for this vulnerable population.

- The communication requirements of older adults should be considered throughout all stages of risk or crisis communication development (before, during, and after an event).

- Older adults and those responsible for their care should be included in the planning process to gain a better understanding of their particular needs and realities. Older adult advocacy groups have the potential to be useful partners in involving older adults in the emergency planning process.

- A common understanding of risk should be communicated and resilience among older adults built by mobilizing individuals and organizations to invest in preparedness.

- The use of clear, simple, easily understandable language in communicating is of particular importance when communicating with older adults.

- Personal preparedness should be encouraged and recommendations tailored to suit the needs and capabilities of older adults.
- Older adults may be less likely to use online sources of information. It is therefore important to continue using traditional modes of communication (eg, print and broadcast media) to maximize coverage for older adults.

- Effective communication can encourage protective health behaviors and in so doing reduce the morbidity and mortality among vulnerable older adults. The need for organizations, such as care homes, to focus on communication during emergency events should therefore be reinforced.

\section{About the Authors}

Department of War Studies (Ms McClelland, Dr Rogers, Mr Tesh, Dr Pearce) and Psychological Medicine (Dr Rubin), King's College London, United Kingdom; and Emergency Response Department, Health Protection Agency, Salisbury, United Kingdom (Dr Amlôt)

Correspondence and reprint requests to Dr. Julia Pearce, Department of War Studies, Strand Campus, London, United Kingdom WC2R 2LS (e-mail: julia. pearce@kcl.ac.uk).

\section{Acknowledgments}

The research was funded by the National Institute for Health Research Health Protection Research Unit (NIHR HPRU) in Emergency Preparedness and Response at King's College London in partnership with Public Health England (PHE). The views expressed are those of the author(s) and not necessarily those of the NHS, the NIHR, the Department of Health, or Public Health England.

Published online: September 20, 2016

\section{REFERENCES}

1. Yazgan IC, Dedeoglu C, Yazgan Y. Disability and post-traumatic psychopathology in Turkish elderly after a major earthquake. Int Psychogeriatr. 2006;18(1):184-187. http://dx.doi.org/10.1017/S1041610206273472.

2. Strug DL, Mason SE, Auerbach C. How older Hispanic immigrants in New York City cope with current traumatic stressors: practice Implications. J Gerontol Soc Work. 2009;52(5):503-516. http://dx.doi. org/10.1080/01634370902983195.

3. Suar D, Mishra S, Khuntia R. Placing age differences in the context of the Orissa Supercyclone: who experiences psychological distress? Asian J Soc Psychol. 2007;10(2):117-122. http://dx.doi.org/10.1111/j.1467-839X. 2007.00218.x.

4. UK Cabinet Office. Identifying People Who Are Vulnerable in a Crisis. https://www.gov.uk/government/uploads/system/uploads/attachment_data/ file/61228/vulnerable_guidance.pdf. Published February 2008. Accessed October 30, 2015.

5. UK Department for Environment, Food \& Rural Affairs. The National Adaptation Programme: Making the country resilient to a changing climate. https:/www.gov.uk/government/uploads/system/uploads/attachment_ data/file/209866/pb13942-nap-20130701.pdf. Published July 2013. Accessed October 30, 2015.

6. US Department of Health and Human Services. Special Populations: Emergency and Disaster Preparedness. http://sis.nlm.nih.gov/outreach/ specialpopulationsanddisasters.html. Last modified December 8, 2014. Accessed August 25, 2015.

7. WHO. Environmental health in emergencies. Vulnerable groups. http:// www.who.int/environmental_health_emergencies/vulnerable_groups/en/. Published 2015. Accessed August 25, 2015.

8. Adams V, Kaufman SR, van Hattum T, et al. Aging disaster: mortality, vulnerability, and long-term recovery among Katrina survivors. Med Anthropol. 2011;30(3):247-270. http://dx.doi.org/10.1080/01459740. 2011.560777. 
9. Ichiseki H. Features of disaster-related deaths after the Great East Japan Earthquake. Lancet. 2013;381(9862):204. http://dx.doi.org/10.1016/ S0140-6736(13)60091-4.

10. UK Civil Contingencies Act 2004. http://www.legislation.gov.uk/ukpga/ 2004/36/contents. Accessed October 30, 2015.

11. Cracknell R. The ageing population. Key Issues for the New Parliament 2010. http://www.parliament.uk/documents/commons/lib/research/key_ issues/Key-Issues-The-ageing-population2007.pdf. Accessed March 26, 2015.

12. United Nations. World Population Ageing: 1950-2050. http://www.un. org/esa/population/publications/worldageing19502050/. Published 2001. Accessed August 25, 2015.

13. NHS. Who should have the flu jab? NHS website. http://www.nhs.uk/ Conditions/vaccinations/Pages/who-should-have-flu-vaccine.aspx. Last modified 11 February 2015. Accessed March 16, 2015.

14. Public Health England. Heatwave Plan for England 2013. https://www. gov.uk/government/uploads/system/uploads/attachment_data/file/201039/ Heatwave-Main_Plan-2013.pdf. Accessed March 26, 2015.

15. OECD. Recommendation of the Council on the Governance of Critical Risks. Paris, France: OECD; 2014

16. UK Cabinet Office. Strategic National Framework on Community Resilience. https://www.gov.uk/government/uploads/system/uploads/ attachment_data/file/60922/Strategic-National-Framework-on-CommunityResilience_0.pdf. Published March 2011. Accessed March 24, 2016.

17. HelpAge International. Disaster Resilience in an Aging World: How to Make Policies and Programmes Inclusive of Older People. London: HelpAge International; 2014.

18. Claver M, Dobalian A, Fickel JJ, et al. Comprehensive care for vulnerable elderly veterans during disasters. Arch Gerontol Geriatr. 2013;56(1):205-213. http://dx.doi.org/10.1016/j.archger.2012.07.010.

19. Rosenkoetter MM, Covan EK, Cobb BK, et al. Perceptions of older adults regarding evacuation in the event of a natural disaster. Public Health Nurs. 2007;24(2):160-168. http://dx.doi.org/10.1111/j.1525-1446. 2007.00620.x.

20. Ardalan A, Mazaheri M, Naieni KH, et al. Older people's needs following major disasters: a qualitative study of Iranian elders' experiences of the Bam Earthquake. Ageing Soc. 2010;30(1):11-23. http://dx.doi.org/10.1017/S0144686X09990122.

21. Rozenblat S, Iecovich E. Sense of threat and coping strategies of Israeli older adults during a military operation. Eur J Ageing. 2013;10(1):61-70. http://dx.doi.org/10.1007/s10433-012-0249-1.

22. Henderson TL, Roberto KA, Kamo Y. Older adults' responses to Hurricane Katrina daily hassles and coping strategies. J Appl Gerontol. 2010;29(1):48-69. http://dx.doi.org/10.1177/0733464809334287.

23. Chaitin J, Sternberg R, Arad H, et al. "I may look 75, but I'm really a pioneer": concept of self and resilience among Israeli elder adults living in a war zone. J Happiness Stud. 2013;14(5):1601-1619. http://dx.doi.org/ 10.1007/s10902-012-9398-3.

24. Sakauye KM, Streim JE, Kennedy GJ, et al. AAGP position statement: disaster preparedness for older americans: critical issues for the preservation of mental health. Am J Geriatr Psychiatry. 2009;17(11): 916-924. http://dx.doi.org/10.1097/JGP.0b013e3181b4bf20.

25. Ticehurst S, Webster RA, Carr VJ, et al. The psychosocial impact of an earthquake on the elderly. Int $J$ Geriatr Psychiatry. 1996;11(11): 943-951. http://dx.doi.org/10.1002/(SICI)1099-1166(199611)11:11<943:: AID-GPS412 > 3.0.CO;2-B.

26. Goenjian AK, Najarian LM, Pynoos RS, et al. Posttraumatic stress disorder in elderly and younger adults after the 1988 earthquake in Armenia. Am J Psychiatry. 1994;151(6):895-901. http://dx.doi.org/ 10.1176/ajp.151.6.895

27. Kato H, Asukai N, Miyake $\mathrm{Y}$, et al. Post-traumatic symptoms among younger and elderly evacuees in the early stages following the 1995 Hanshin-Awaji Earthquake in Japan. Acta Psychiatr Scand. 1996;93(6): 477-481. http://dx.doi.org/10.1111/j.1600-0447.1996.tb10680.x.

28. Kleinpeter MA. Disaster preparedness of dialysis patients for Hurricanes Gustav and Ike 2008. Adv Perit Dial. 2009;25:62-67.
29. Fernandez LS, Byard D, Lin C-C, et al. Frail elderly as disaster victims: emergency management strategies. Prehosp Disaster Med. 2002;17(02): 67-74. http://dx.doi.org/10.1017/S1049023X00000200.

30. Phifer JF, Norris FH. Psychological symptoms in older adults following natural disaster: nature, timing, duration, and course. J Gerontol. 1989; 44(6):S207-S212. http://dx.doi.org/10.1093/geronj/44.6.S207.

31. Monahan K, Lurie A. Reactions of senior citizens to 9/11: exploration and practice guidelines for social workers. Soc Work Health Care. 2007; 45(1):33-47. http://dx.doi.org/10.1300/J010v45n01_03.

32. Kohn R, Levav I, Garcia ID, et al. Prevalence, risk factors and aging vulnerability for psychopathology following a natural disaster in a developing country. Int J Geriatr Psychiatry. 2005;20(9):835-841. http://dx.doi.org/10.1002/gps.1369.

33. Shrira A, Palgi Y, Hamama-Raz Y, et al. Previous exposure to the World Trade Center terrorist attack and posttraumatic symptoms among older adults following Hurricane Sandy. Psychiatry. 2014;77(4):374-385. http://dx.doi.org/10.1521/psyc.2014.77.4.374.

34. Pietrzak RH, Southwick SM, Tracy M, et al. Posttraumatic stress disorder, depression, and perceived needs for psychological care in older persons affected by Hurricane Ike. J Affect Disord. 2012;138(1-2):96-103. http://dx.doi.org/10.1016/j.jad.2011.12.018.

35. Brown LM, Cohen D, Kohlmaier JR. Older adults and terrorism. In: Bongar B, Brown LM, Beutler LE, eds. Psychology of Terrorism. New York: Oxford University Press; 2007:288-310.

36. Christensen JJ, Richey ED, Castaneda H. Seeking safety: predictors of hurricane evacuation of community-dwelling families affected by alzheimer's disease or a related disorder in south Florida. Am J Alzheimers Dis Other Demen. 2013;28(7):682-692. http://dx.doi.org/10.1177/ 1533317513500837.

37. Kamo Y, Henderson TL, Roberto KA. Displaced older adults' reactions to and coping with the aftermath of Hurricane Katrina. J Fam Issues. 2011;32(10):1346-1370. http://dx.doi.org/10.1177/0192513X11412495.

38. Behr JG, Diaz R. Disparate health implications stemming from the propensity of elderly and medically fragile populations to shelter in place during severe storm events. J Public Health Manag Pract. 2013;19(5): S55-S62. http://dx.doi.org/10.1097/PHH.0b013e318297226a.

39. Nishikiori N, Abe T, Costa DGM, et al. Who died as a result of the tsunami? Risk factors of mortality among internally displaced persons in Sri Lanka: a retrospective cohort analysis. BMC Public Health. 2006;6:73.

40. Hall N. Older persons in disasters and emergencies: the overlapping mental and physical health issues. In: Toner JA, Mierswa TM, Howe JL, eds. Geriatric Mental Health Disaster and Emergency Preparedness. New York: Springer Publishing Company; 2010:31-46.

41. Langan JC, Palmer JL. Listening to and learning from older adult Hurricane Katrina survivors. Public Health Nurs. 2012;29(2):126-135. http://dx.doi.org/10.1111/j.1525-1446.2011.00996.x.

42. Mitani S, Kako M, Mayner L. Medical relief for the 2011 Japan earthquake: a nursing account. Nurs Health Sci. 2014;16(1):26-30.

43. World Health Organization. Older People in Emergencies: Considerations for Action and Policy Development. http://www.who.int/ageing/ publications/Hutton_report_small.pdf Published 2008. Accessed March 05, 2016.

44. Christensen JJ, Brown LM, Hyer K. A haven of last resort: the consequences of evacuating Florida nursing home residents to nonclinical buildings. Geriatr Nurs (Minneap). 2012;33(5):375-383. http://dx.doi.org/10.1016/j.gerinurse.2012.03.014.

45. Parmar P, Arii M, Kayden S. Learning from Japan: strengthening US emergency care and disaster response. Health Aff. 2013;32(12): 2172-2178. http://dx.doi.org/10.1377/hlthaff.2013.0704.

46. Brown LM, Dosa DM, Thomas K, et al. The effects of evacuation on nursing home residents with dementia. Am J Alzheimers Dis Other Demen. 2012;27(6):406-412. http://dx.doi.org/10.1177/1533317512454709.

47. Jain SL, Kamimoto L, Bramley AM, et al. Hospitalized patients with 2009 H1N1 Influenza in the United States, April-June 2009. $N$ Engl J Med. 2009;361(20):1935-1944. http://dx.doi.org/10.1056/ NEJMoa0906695. 
48. Katz JM, Plowden J, Renshaw-Hoelscher M, et al. Immunity to influenza: the challenges of protecting an aging population. Immunol Res. 2004; 29(1-3):113-124. http://dx.doi.org/10.1385/IR:29:1-3:113.

49. Deans GD, Stiver HG, McElhaney JE. Influenza vaccines provide diminished protection but are cost-saving in older adults. J Intern Med. 2010;267(2):220-227. http://dx.doi.org/10.1111/j.1365-2796.2009.02201.x.

50. Lang PO, Samaras D, Samaras N, et al. Influenza vaccination in the face of immune exhaustion: is herd immunity effective for protecting the elderly? Influenza Res Treat. 2011;2011:419216. http://dx.doi.org/10.1155/ 2011/419216.

51. Rogers MB, Pearce JM. Risk communication, risk perception and behaviour as foundations of effective national security practices. In: Akhgar B, Yates S. Strategic Intelligence Management. Amsterdam: Butterworth-Heinemann; 2013:66-74.

52. Rogers MB, Pearce JM. The psychology of crisis communication. In: Loffelholz M, Schwarz A, Seeger MW. The Handbook of International Crisis Communication Research. Hoboken, NJ: John Wiley \& Sons; 2016:34-44. 10.1002/9781118516812.ch4.

53. Reynolds B. Crisis and Emergency Risk Communication: Pandemic Influenza. Atlanta, GA: Centers for Disease Control and Prevention; 2007.

54. Heath RL, O'Hair HD. The significance of crisis and risk communication. In: Heath RL, O'Hair HD. Handbook of Risk and Crisis Communication. New York: Routledge; 2010:5-30

55. Centers for Disease Control and Prevention. Crisis and Emergency Risk Communication. Atlanta, GA: Centers for Disease Control and Prevention; 2014.

56. Glik DC. Risk communication for public health emergencies. Annu Rev Public Health. 2007;28(1):33-54. http://dx.doi.org/10.1146/annurev. publhealth.28.021406.144123.

57. MacDonald L, Cairns G, Angus K, et ak. Promotional communications for influenza vaccination: a systematic review. J Health Commun. 2013; 18(12):1523-1549. http://dx.doi.org/10.1080/10810730.2013.840697.

58. Prati G, Pietrantoni L, Zani B. Influenza vaccination: the persuasiveness of messages among people aged 65 years and older. Health Commun. 2012;27(5):413-420. http://dx.doi.org/10.1080/10410236. 2011.606523.

59. Greenberg M. We need risk communication and perception research focused on flood risks for senior citizens. Risk Anal. 2013;33(1):52-53. http://dx.doi.org/10.1111/j.1539-6924.2012.01940.x.

60. Al-rousan TM, Rubenstein LM, Wallace RB. Preparedness for natural disasters among older US adults: a nationwide survey. Am J Public Health. 2014;104(3):506-511. http://dx.doi.org/10.2105/AJPH.2013.301559.
61. Loke AY, Lai CK, Fung OWM. At-home disaster preparedness of elderly people in Hong Kong. Geriatr Gerontol Int. 2012;12(3):524-531. http://dx.doi.org/10.1111/j.1447-0594.2011.00778.x.

62. Centers for Disease Control and Prevention Healthy Aging Program. Disaster planning tips for older adults and their families. http://www.cdc. gov/aging/pdf/disaster_planning_tips.pdf. Accessed March 6, 2016.

63. Rosenkoetter MM, Covan EK, Bunting S, et al. Disaster evacuation: an exploratory study of older men and women in Georgia and North Carolina. J Gerontol Nurs. 2007;33(12):46-54.

64. Rogers MB, Amlot R, Rubin GJ, et al. Mediating the social and psychological impacts of terrorist attacks. Int Rev Psychiatry. 2007;19(3): 279-288. http://dx.doi.org/10.1080/09540260701349373.

65. Heppenstall CP, Wilkinson TJ, Hanger HC, et al. Impacts of the emergency mass evacuation of the elderly from residential care facilities after the 2011 Christchurch Earthquake. Disaster Med Public Health Prep. 2013;7(4):419-423. http://dx.doi.org/10.1017/dmp.2013.47.

66. Becker SM. Emergency communication and information issues in terrorist events involving radioactive material. Biosecurity and Bioterrorism: Biodefence, Strategy, Practice and Science. 2004;2(3):195-207. http://dx.doi.org/10.1089/bsp.2004.2.195.

67. Neuhauser L, Ivey SL, Huang D, et al. Availability and readability of emergency preparedness materials for deaf and hard-of-hearing and older adult populations: issues and assessments. PLoS One. 2013;8(2):e55614. http://dx.doi.org/10.1371/journal.pone.0055614.

68. Office for National Statistics. Internet Users, 2015. http://www.ons.gov. uk/ons/dcp171778_404497.pdf. Published May 22, 2015. Accessed October 29, 2015.

69. Silver A, Conrad C. Public perception of and response to severe weather warnings in Nova Scotia, Canada. Meteorol Appl. 2010;17(2):173-179. http://dx.doi.org/10.1002/met.198.

70. Johnson A, Howe JL, McBride MR, et al. Bioterrorism and emergency preparedness in aging (BTEPA): HRSA-funded GEC collaboration for curricula and training. Gerontol Geriatr Educ. 2006;26(4):63-86. http://dx.doi.org/10.1300/J021v26n04_06.

71. Huerta F, Horton R. Coping behavior of elderly flood victims. Gerontologist. 1978;18(6):541-546. http://dx.doi.org/10.1093/geront/18.6.541.

72. Seeger MW. Best practices in crisis communication: an expert panel process. J Appl Commun Res. 2006;34(3):232-244. http://dx.doi.org/ 10.1080/00909880600769944.

73. Lock S, Rubin GJ, Murray V, et al, Secondary stressors and extreme events and disasters: a systematic review of primary research from 2010. 2011. PLOS Curr Disasters. 2012 Oct 29. doi: 10.1371/currents.dis. a9b76fed1b2dd5c5bfcfc13c87a2f24f. 